\title{
Norois
}

Environnement, aménagement, société

$231 \mid 2014$

Les modes d'habiter à l'épreuve de la durabilité

\section{Systèmes de valeurs vs pragmatisme dans les choix de pratiques spatiales : la place de la durabilité}

systems of values vs pragmatism in the choice of spatial practices: the role of sustainability

Denis Martouzet

\section{OpenEdition}

Journals

Édition électronique

URL : http://journals.openedition.org/norois/5119

DOI : $10.4000 /$ norois. 5119

ISBN : 978-2-7535-3599-2

ISSN : 1760-8546

Éditeur

Presses universitaires de Rennes

Édition imprimée

Date de publication : 30 septembre 2014

Pagination : 125-136

ISBN : 978-2-7535-3465-0

ISSN : 0029-182X

Référence électronique

Denis Martouzet, « Systèmes de valeurs vs pragmatisme dans les choix de pratiques spatiales : la place de la durabilité », Norois [En ligne], 231 | 2014, mis en ligne le 30 juin 2016, consulté le 01 mai 2019. URL : http://journals.openedition.org/norois/5119; DOI : 10.4000/norois.5119

(c) Tous droits réservés 


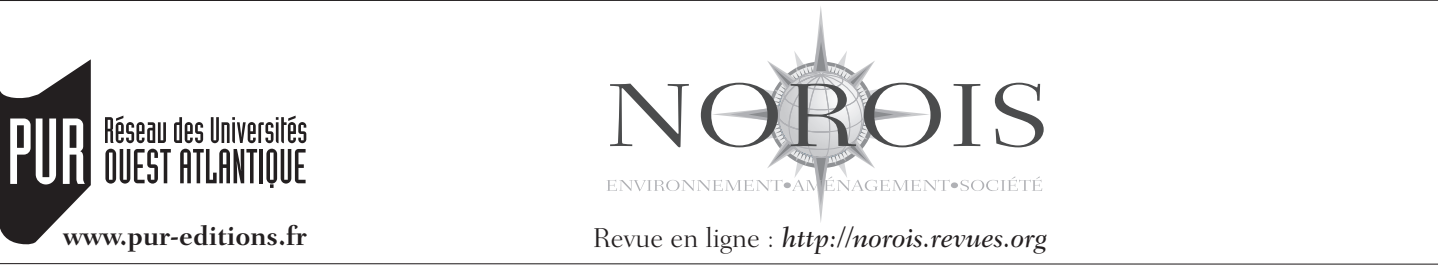

\title{
Systèmes de valeurs vs pragmatisme dans les choix de pratiques spatiales : la place de la durabilité
}

\author{
Systems of Values vs Pragmatism in the Choice of Spatial Practices: \\ The Role of Sustainability
}

\author{
Denis Martouzet
}

\begin{abstract}
CITERES - UMR 7324 CNRS/Université de Tours, 33-35, allée Ferdinand-de-Lesseps, 37200 Tours, France
(denis.martouzet@univ-tours.fr)
\end{abstract}

Résumé : Dans une recherche menée pour le compte du PUCA, l'analyse de matériaux discursifs recueillis lors d'enquêtes révèle que la durabilité se dégage, pour les individus, comme valeur, que ce soit dans une optique kantienne, absolutiste et déontologique ou une optique relativiste et conséquentialiste. Autour de ces deux conceptions de la valeur, cet article retrace les façons dont les individus, lorsqu'ils sont invités à évoquer leur mobilité et plus largement leurs pratiques spatiales, évoquent, mobilisent, évacuent, nient la notion de durabilité. Les individus de notre échantillon sont suivis par GPS pendant une semaine ordinaire. Puis, lors d'un entretien, ils commentent le tracé de leurs déplacements et expliquent et justifient leurs choix. Cinq grandes figures permettent de différencier ces individus qui négocient avec cette valeur, soit par conciliation, soit par compensation.

\begin{abstract}
In a research conducted for PUCA (inter-ministerial Plan for Urban Development Construction and Architecture), the analysis of discursive materials, gathered during investigations, reveals that sustainability emerges as a value for people, whether in a Kantian, absolutist and deontological perspective or in a relativistic and consequentialist way. Around these two conceptions of value, this paper tells how individuals, when they are invited to discuss their mobility and their wider spatial practices, suggest, mobilize, evacuate or deny the notion of sustainability. In our sample, individuals are monitored by GPS during a regular week. Then, during an interview, they comment the tracks of their movements and explain and justify their choices. Five great figures can be differentiated in this sample of individuals who deal with this value, either by conciliation or by compensation.
\end{abstract}

Mots clés : Durabilité - mode d'habiter - mobilité - valeur - pragmatisme - conciliation - compensation

Keywords: Sustainability, way of living, mobility, value, pragmatism, conciliation, compensation

Dans le cadre d'une recherche menée pour le compte du PUCA, dans un programme portant sur la durabilité des espaces périurbains ${ }^{1}$, une série

1. «PERIVIA - Le périurbain à l'épreuve des modèles d'habiter, la viabilité périurbaine entre théorie(s) et pratique(s) », recherche financée par le PUCA, d'enquêtes a été réalisée. L’objectif, au-delà du motif premier d'évaluation de ce géotype (Lévy J., 2003, p. 412-413), est de saisir les motivations pro-

appel à projets « La mobilité et le périurbain à l’impératif de la ville durable : ménager les territoires de vie des périurbains ». Nov. 2009-Nov. 2011. 
fondes des individus lorsqu'ils procèdent à des choix spatiaux, en ce qui concerne la localisation de leurs activités ${ }^{2}$ et leurs déplacements, considérés comme moyen d'ajustement global de celles-ci. Nous avons mis l'accent sur la mobilité, dont on connaît l'importance sociétale (Urry, 2005; Stock, 2005, 2006) et individuelle actuelle (Ramadier, 2007, 2009) en termes d'enjeux aux différentes échelles sociales. Elle est ici considérée comme constitutive de l'habiter, au sens phénoménologique du terme (Paquot, Lussault, Younès, 2007) : elle en est la dimension mobile, par complémentarité avec la «lieuité » qui en est la dimension statique. Les liens entre ancrage et mobilité (Rémy, 1996; Orfeuil, 1998; Carpentier, 2007a, 2007b ; Feildel et Martouzet, 2012; Kaufmann, 1999, 2008) servent de cadre général d'analyse des motivations associées aux choix des individus.

Les pratiques spatiales ainsi observées sont questionnées, principalement, par la notion de durabilité. Il s'est agi de saisir, parmi les éléments mobilisés par des personnes pour justifier (Thévenot, 1996) de leur mobilité, ce qui relève de valeurs et, parmi celles-ci, celle de durabilité.

Le périurbain, largement décrié sur le plan de sa durabilité, opposé à la ville qui par certains aspects apparaît comme un modèle, est pourtant maintenant analysé de façon plus nuancée (Cailly, 2007, 2008; Dodier, 2007, 2009), y compris en termes prospectifs (Cordobes et al., 2010; Roux et Vanier, 2008). Le terrain périurbain d'application de cette recherche, celui autour de la ville de Tours, est structuré par un réseau viaire en étoile à partir de la ville centre, mais aussi par des possibilités de déplacements en transports en commun (lignes de la SNCF, cars SNCF, cars du Conseil Général et, plus localement, bus urbains) et en transports dits doux. Au total, la possibilité de choix autorisée par cette offre a permis - plus que dans d'autres types d'espaces qui n'offrent pas d'alternatives à la voiture ou rendent les choix alternatifs peu coûteux (modes doux d'utilisation aisée pour des citadins ayant toutes leurs activités à proximité) - de mettre l'individu enquêté, face à un éventail de possibilités très ouvert, en situation de se confronter à ses

2. Il s'agit, plus exactement encore, des choix relatifs au couple formé d'une activité et de sa localisation, l'activité elle-même renvoyant à des localisations parmi lesquelles choisir et les localisations possibles renvoyant à la possibilité ou non (et le coût global) de pratiquer l'activité. propres choix, le renvoyant à ses motivations et à ses valeurs.

L'optique choisie ici, celle d'une entrée par la place de la valeur de durabilité dans les motivations d'actions (ici, plus précisément, les actions de déplacement ou les activités nécessitant déplacement), renvoie aux débats relatifs à la durabilité, d'une part, et à ceux relatifs à la nature et au statut mêmes de la valeur (non celle de durabilité mais de la valeur sur un plan notionnel), d'autre part. Notons donc que nous nous situons ici dans la perspective d'une analyse d'ordre philosophique. Cette précision vise à clarifier les deux points suivants. Premièrement, les valeurs sont à appréhender ici en tant qu'elles ont été mobilisées explicitement ou implicitement dans les discours recueillis par les personnes amenées à justifier certains de leurs choix. Deuxièmement, il ne s'agit pas ici d'examiner les modalités de la construction de ces valeurs, que ce soit dans leur dimension sociale ou leur dimension individuelle, il s'agit simplement de questionner le résultat, non définitif au moment de l'entretien, de cette construction. Cela renvoie à la valeur que l'individu confère à ces/ses valeurs : se situe-t-il dans un cadre kantien déontologique ou conséquentialiste relativiste? Dit autrement, les valeurs sont-elles des objets construits préalablement à l'action et aux discours qui, alors en sont nécessairement teintés, ou se construisent-elles, se négocient-elles dans l'action ou encore dans le discours? Les valeurs pensées celles dont l'individu est intimement convaincu du bien-fondé - résistent-elles à l'épreuve des faits? Ce faisant, le processus de construction individuelle ou sociale des valeurs n'est pas abordé, c'est le résultat, au moment de l'entretien, de cette construction qui est pris en compte, laissant ainsi de côté la dimension individuelle et sociale de la construction des valeurs. De ce fait, si l'individu examiné est bien inséré de multiples façons synchrones et successives dans le corps social, il est ici, de façon volontairement isolée, notre échelle sociale qui synthétise toute influence sociale ou autre (comme le moment de l'entretien est l'échelle temporelle qui condense l'ensemble des processus qui ont conduit au contenu de ce moment) d'appréhension de la question éthique de la durabilité en lien avec le moment de l'enquête. Cet individu est aussi le « lieu » qui nous fournit via ses discours le matériau de l'analyse menée. D'autres approches sont pos- 
sibles, institutionnelles (Villalba, Lejeune, 2010) ou socio-économiques (Keramidas et al., 2010) qui évacuent généralement l'approche individu-centrée.

Nous examinons les deux conceptions majeures de la valeur de façon à clarifier plus encore notre positionnement et nos questionnements, et la notion de durabilité, avant d'aborder les aspects méthodologiques. Par la suite, des résultats sont donnés sous la forme de figures de personnes, distinguées par la manière dont elles pratiquent l'espace, par la mobilisation qu'elles font ou non de la durabilité et du statut qu'elles lui confèrent. Ces figures sont ensuite subsumées par l'entremise de la durabilité comme valeur et des modes de "faire avec » cette valeur dans l'épreuve des faits.

\section{Positionnement, Problématique ET ÉTAT DE L'ART : LA DURABILITÉ COMME VALEUR}

«Lorsque, malgré tout, on associe une valeur à quelque chose (une fin, un comportement ou un individu), on ne sait pas si cette valeur appartient intrinsèquement à l'être en question ou si elle lui est, comme on dit, "attribuée" par une décision subjective ou extérieure »(Pharo, 2007, p. 836). Pharo indique ainsi les deux grands courants de la philosophie des valeurs. L'un relève d'une tradition kantienne (Kant, 1988), que l'on peut qualifier d'absolutiste ou déontologique, l'autre d'une tradition relativiste et conséquentialiste, notamment chez les utilitaristes au premier rang desquels on peut positionner Jeremy Bentham (1970). De façon schématique, dans le cadre d'une réflexion éthique ${ }^{3}$, un acteur absolutiste agit par principe et par devoir (deontos) alors que l'acteur relativiste admet que l'ensemble des valeurs forment système et sont en concurrence : il agit alors selon l'évaluation qu'il fait des conséquences potentielles qu'il connaît de son acte. Cela n'est pas sans renvoyer à Weber (1959), qui distingue éthique de la responsabilité et éthique de la conviction.

Cependant, et même si les résultats que nous avons obtenus sur la question de la durabilité des pratiques spatiales renvoient partiellement à cette partition, celle-ci - qui pose les bases d'une discus-

\footnotetext{
3. Les valeurs ne relèvent pas uniquement de l'éthique, il existe des valeurs esthétiques, politiques, économiques, sociales, environnementales (Ogien, 1996).
}

sion sur la valeur des valeurs - ne permet pas de décrire l'intégralité de ces résultats. Il est utile, pour la compréhension générale du propos, d'y ajouter l'examen du rapport entre normes et valeurs. En une première approche, on peut évoquer la possibilité de normes qui seraient sociales et de valeurs qui seraient individuelles (éventuellement socialement partagées). Les premières s'imposent à l'individu qui, en retour, par sa capacité d'autonomie est capable de s'y conformer ou non, notamment en lien avec ses valeurs individuelles, qui peuvent s'y opposer ou se confondre avec elles. Plus précisément, la valeur se définit par sa double orientation ou sa double « direction d'ajustement » (Livet, 2006; Jaquet, 2014), de l'individu au monde puis du monde à l'individu. Face à un objet, l'esprit, pour reprendre le terme utilisé par Ogien (1996), porte une évaluation sur cet objet. Il lui attribue une valeur et ce faisant l'objet renvoie une injonction à l'esprit, qui ainsi envisage de modifier l'objet (ou de le maintenir si l'évaluation est positive). De façon inverse dans le cas de la norme, c'est d'abord l'objet qui s'impose à l'esprit, qui ensuite évalue l'objet, mais cette évaluation est teintée du premier mouvement. «Les normes suivent les directions d'ajustement dans l'ordre inverse des valeurs » (Livet, 2006, p. 35). Ainsi, normes et valeurs se différencient mais se complètent l'une l'autre par des mouvements d'aller et retour.

Norme pour certains, valeur pour d'autres, la durabilité, l'émergence et la diffusion de la notion, les débats qui ont découlent, les enjeux qui y sont associés amènent à changer le regard que nous portons sur le monde et sur nous-mêmes, nos pratiques et nos attitudes, nos décisions et nos actions. Côté norme, la durabilité comme injonction se décline en une série de questionnements et de normes techniques ou politico-administratives se traduisant par un vaste cahier des charges appliqué collectivement (participation, éco-construction, agenda $21 \ldots$ ), à différentes échelles spatiales, tous domaines et toutes approches confondues. Cette norme générale ou cet ensemble de normes particulières ne cesse de s'accroître et se formule, implicitement ou explicitement, par des injonctions sociétales qui s'imposent à l'individu. Mais nous formulons, en parallèle, l'hypothèse que cette durabilité ne relève pas exclusivement d'une stricte application normative. Elle est aussi du ressort de l'individu 
qui, en intégrant ce paramètre comme une dimension culturelle parmi d'autres et non plus en tant que contrainte, si ce n'est éventuellement comme auto-contrainte, confère à la durabilité le statut de valeur, soit comme principe, soit comme élément d'une éthique conséquentialiste.

La notion de durabilité, par le flou qui lui est inhérent et par la multiplicité des usages qui en sont faits, fait débat et amène des positions exacerbées. Navez-Bouchanine (2007) parle de «leurre scientifique ». Mathieu et Guermond l'envisagent comme utopie politique aussitôt mise à mal : « Ne s'agit-il donc pas d'un habillage des politiques non résolues sous les pseudo-habits neufs d'une prise de conscience mondiale, d'un recyclage des anciens objectifs et des pratiques politiques donnant l'illusion de la conciliation entre pauvreté, développement du libéralisme économique et préservation des ressources renouvelables et de la biodiversité? » (2005, p. 15). Bosc (2003) y décèle la forme néolibérale d'une rhétorique environnementaliste. D'autres y voient ce qui conduirait à un « tournant urbanistique » (Emelianoff, 2007) ou à un « nouvel urbanisme » (Da Cunha, 2005). Enfin, d'autres encore, cherchent à classer les différentes approches du développement durable. Par exemple, Mancebo (2007) distingue la durabilité forte de la durabilité faible, Joëlle Salomon Cavin et Dominique Bourg (2010) les approches intégrées des approches technologiques. Ce qu'il convient de retenir ici est l'extrême difficulté - pour ne pas dire l'impossibilité - de saisir une définition qui ferait unanimité, associée à l'ampleur du champ sémantique de la notion. Aussi, dans le cadre des entretiens menés dans les travaux restitués ici, il n'a pas pu être envisagé techniquement de fournir cette précision aux individus enquêtés par entretien. Par ailleurs, procéder ainsi aurait étouffé toute spontanéité de la part de l'interviewé qui aurait cherché à répondre à une certaine idée de la durabilité. Aussi, finalement, avons-nous admis ce que les personnes elles-mêmes mobilisent, généralement de façon implicite, sous ce terme.

\section{SAISIR LES MOTIVATIONS DERRIÈRE/ PAR LES JUSTIFICATIONS : ASPECTS MÉTHODOLOGIQUES}

Quelle que soit la conception que l'on retienne de la valeur, elle est, au même titre que d'autres moti- vations, non seulement ce qui fait agir, mais aussi ce qui fait agir dans une certaine direction. On ne peut y avoir accès qu'indirectement, par le biais de l'observation des pratiques qui en seraient révélatrices ou par le biais des discours. De là, découlent les aspects méthodologiques.

Plusieurs raisons rendent difficile la saisie des valeurs portées par les individus (et qui d'ailleurs portent ceux-ci). Tout d'abord, sur le plan temporel, elles structurent le long terme de l'échelle biographique et, dans le même temps, elles sont également mobilisées dans le court terme, voire dans le vif de l'instant présent pour l'action et pour l'évaluation, dans les différentes situations éprouvées. Par ailleurs, au-delà même de la difficulté à définir le terme de valeur, chaque valeur a, par le caractère incomplet et inexact du vocabulaire, une signification aux contours flous. Il en est ainsi, par exemple, de la durabilité. La mise en ouvre d'une action supportée par une valeur reste donc problématique, dans la mesure où elle ne s'inscrit pas nécessairement dans le cadre d'une valeur qui serait strictement appliquée. Une action déconnectée de tout intérêt individuel, par exemple, est difficile à envisager, du moins, peut-on douter de son existence en situation. En tant qu'un des moteurs de l'agir, une valeur peut entrer en concurrence avec d'autres valeurs ou motivations, ou encore, s'y associer. Ainsi, toute action est prise dans un réseau dynamique, évolutif et flou de motivations diverses qui se renforcent ou se nuancent, mais duquel l'individu fait nécessairement une synthèse conciliatrice, concrétisée dans l'action même. Il est donc malaisé d'isoler une valeur, même si on peut estimer sa place dans une hiérarchie des motivations.

D'autre part, une valeur est aussi une mise en jeu de l'individu par lui-même et pour lui-même. Aussi, pourra-t-il, à ses propres yeux ou en situation de relation à autrui, préférer justifier son action par une motivation valorisante plutôt que par une autre, selon certains critères renvoyant eux-mêmes à des valeurs, à des normes... Plus encore, la situation d'enquête en vis-à-vis, du fait du regard de l'autre, exacerbe cet enjeu. On peut ainsi toujours douter que les valeurs mobilisées dans les discours - ou même l'introspection - soient le reflet exact de celles qui ont été mobilisées dans l'action.

N'ayant pas accès directement aux valeurs (par nature non observables), deux médias peuvent être 
utilisés. D’une part, les pratiques en tant qu'elles sont observables et parce qu'on les suppose soustendues possiblement par des valeurs parmi d'autres motivations, qu'elles révèlent en partie. D'autre part, les discours, pour les mêmes raisons, car discourir est un mode d'agir, au-delà des effets rhétoriques ou de la nécessité de justifier ses actions. Le couplage, dans l'analyse, des résultats relatifs aux pratiques et aux dires permet en partie d'atteindre les valeurs, de les saisir. S'il ne permet pas l'accès aux raisons de la survalorisation de telle valeur par tel individu ou tel type de situation, il est néanmoins possible de décrire les réseaux de motivations de façon à construire des figures.

La méthode de saisie des valeurs mobilisées dans l'action - notamment la valeur de durabilité parmi d'autres - repose ici sur le couplage d'une mesure aussi précise et objective que possible des déplacements effectifs et du recueil de l'éprouvé subjectif de ces déplacements, c'est-à-dire la mobilité de l'individu. Par mobilité, nous entendons ainsi la signification pleine et entière donnée aux déplacements par ceux qui les effectuent (Bailleul et Feildel, 2011; Martouzet et al., 2010). Dans cette signification entrent bien évidemment les causes et objectifs de ces déplacements. Entrent également les modalités matérielles, spatiales et temporelles de ceux-ci. Enfin et surtout, intervient le système représentationnel qu'a l'individu de la situation dans laquelle il se trouve engagé. Au-delà des motifs enfin, la décision de se déplacer ou non, et selon quelles modalités, ainsi que les motivations de ces arbitrages sont considérées.

La mobilité est définie différemment selon les auteurs (Aubertel et Bonnet, 2006; Bassand et Brulhardt, 1980 ; Courgeau, 1988; Dureau et Lévy, 2002; Marzloff et Kaplan, 2009; Montulet, 1998). De notre côté, nous considérons que l'habiter est la signification globale de la spatialité d'un individu. Alors l'habiter est à la mobilité ce que la spatialité est aux déplacements, ou, dit autrement, l'habiter est à la spatialité ce que la mobilité est aux déplacements (EhEA, 2008). Nous sommes bien, dans le couplage entre une approche quantitative objective et une approche qualitative rendant compte du subjectif, dans un rapport entre signifié et signifiant. La difficulté réside dans l'interprétation du signifié à partir du signifiant. Aussi, l'approche quantitative n'a-t-elle pas pour objet principal de donner une mesure, des éléments chiffrés (même si ce fut fait et interprété); son caractère objectif - en tout cas proche d'une certaine objectivité et pensé comme tel par les personnes interviewées - donne à l'entretien un caractère d'épreuve de vérité.

On a ainsi confié à 37 personnes volontaires un boitier GPS pendant une semaine, qualifiée par ellesmêmes d'ordinaire, c'est-à-dire mêlant des déplacements entre le domicile, le lieu de travail, les lieux de consommation courante et de loisir réguliers, les lieux de scolarisation des enfants. Ces 37 GPS ont été programmés pour fonctionner de 5 heures du matin à 23 heures (minuit le samedi) de façon à ce que la personne n'ait pour obligation que de le recharger électriquement et de l'avoir constamment sur elle, afin de recueillir avec la plus grande exactitude possible l'intégralité de ses déplacements. Aucune indication n'était donnée quant aux objectifs de la recherche. On connaît les limites de ce type de recueil d'information : oublis de l'avoir sur soi, « oublis volontaires », déplacements modifiés par le port même du GPS, défaillances techniques et imprécisions (Chardonnel, 2011; Depeau, 2011). Etant donné que le GPS n'avait pas pour but principal la plus grande précision mais plutôt la fourniture d'un matériau (la carte des tracés GPS) de réactivation du discours à visée qualitative, la marge d'erreur associée à la faillibilité du protocole dans sa dimension quantitative n'obère pas la pertinence de l'approche qualitative $^{4}$. La figure 1 (planche VIII, p. 112) présente un exemple de carte des déplacements hebdomadaires d'une des personnes de l'échantillon. Il faut cependant noter que cette carte, présentée à son « auteur » lors de la phase de réactivation de l'entretien, l'était sur écran d'ordinateur dans le but de pouvoir zoomer à sa demande, de choisir le ou les jours pour lesquels afficher les déplacements, etc.

L'échantillon retenu n'a aucune prétention à une représentativité même relative. A été privilégiée la diversité des situations envisageables, en fonction notamment de la localisation du logement et du lieu de travail, de la profession exercée et des horaires qu'elle implique, de la composition de la famille, de l'âge, du genre et des ressources financières. Plus précisément, les 37 personnes interviewées ont un âge compris entre 14 et 74 ans (c'est-à-dire ayant

\footnotetext{
4. C'est surtout l'apparence de précision et d'objectivité qui compte ici car elle met l'interviewé en position de se sentir face à ses pratiques, sans qu'il puisse, d'une façon ou d'une autre, les nier.
} 
une certaine autonomie dans leurs déplacements). Il y 22 femmes et 15 hommes. Ils représentent essentiellement les classes moyennes disposant de ressources leur permettant d'assurer, entre autres, leurs déplacements (19 professions intermédiaires; 6 cadres et professions intellectuelles supérieures; 5 retraités; 3 étudiants; 2 artisans-commerçants; 2 employés). En termes de localisation du lieu de résidence, 16 personnes sont à moins de $10 \mathrm{~km} \mathrm{du}$ centre de Tours, 10 à une distance de ce centre comprise entre 10 et $20 \mathrm{~km}, 6$ entre 20 et $30 \mathrm{~km}$, 19 entre 30 et 40, 2 au-delà, jusqu'à $55 \mathrm{~km}$. Pour le couple lieu de travail/lieu de résidence, quatre catégories ont été distinguées : 20 individus habitent dans le périurbain et travaillent dans l'agglomération de Tours; 11 individus habitent dans le périurbain et travaillent dans le périurbain; 4 habitent l'agglomération de Tours et travaillent dans le périurbain; enfin, 2 habitent dans l'agglomération de Tours et travaillent dans cette agglomération. L'entretien, ouvert et peu directif, a été structuré en trois temps bien distincts, annoncés comme tels à l'interviewé, sans que le contenu du troisième temps soit dévoilé avant la fin du précédent. Le premier, de vingt à quarante minutes, vise à saisir le profil de l'interviewé concernant sa mobilité, ainsi que son habiter, par le récit qu’il fait de son parcours biographique, depuis les plus anciens souvenirs jusqu'au moment de l'enquête : les différents logements occupés, les allers et retours vers l'école puis le collège, etc. jusqu'aux différents lieux de travail, les premiers moyens de transport utilisés, ceux des parents et ceux qui ont suivis, les préférences, les bons et moins bons souvenirs concernant des trajets... Ce premier temps est clos par une synthèse, en quelques phrases de ce parcours, orientée vers les grands traits de la mobilité de l'individu.

Le deuxième temps, plus long, dépassant parfois une heure, mobilise le tracé GPS obtenu, apposé sur le fond Google Earth que l'on peut, à la demande de l'interviewé et/ou selon les besoins liés aux questions abordées, faire changer d'échelle, sur écran d'ordinateur. Cette carte, très précise, est agrémentée d'un tableau recensant tous les déplacements, précisant le point origine, le point destination, la vitesse moyenne sur chaque tronçon, la distance parcourue. De ces données fournies directement par l'appareillage, on peut déduire le mode de déplace- ment et le motif5. L'interviewé est convié à décrire et commenter ses trajets et leurs modalités, se sentant généralement obligé d'en préciser le pourquoi. Un discours purement descriptif n'est pas tenable pour l'enquêté dans ce cadre et une nécessité de justification apparaît : Pourquoi là, à ce moment, de cette manière? Pourquoi ce trajet plutôt qu'un autre pour aller d'un point $\mathrm{A}$ à un point $\mathrm{B}$ ? Pourquoi par tel mode de transport? Pourquoi à cette vitesse?

Au troisième temps, d'environ trente minutes, l'enquêteur demande quelles sont les pratiques que la personne effectue (ou non) qui pourraient, selon elle, être qualifiée de durables, tout champ d'action confondu (dépassant donc le strict plan des déplacements) et ce qu'elle pense de telles pratiques. L'enquêteur dévoile ainsi son objectif de saisie de la durabilité comme élément, éventuellement comme valeur, mobilisé dans l'action. Mais, ce faisant, en plus de dévoiler en partie ce qu'il intègre dans la notion de durabilité, l'enquêté y voit une forme de jugement de valeur : aussi se tient-il prêt à justifier plus encore ses pratiques, ce qui permet de faire émerger ses motivations et valeurs ${ }^{6}$. On peut ainsi estimer l'importance de la place occupée par la durabilité et avoir quelques indices quant au statut qui lui est conféré (valeur, norme intégrée, norme ressentie...). De façon spontanée ou par une relance de l'enquêteur, cela remet en perspective les justifications apportées dans le deuxième temps aux déplacements effectués : nuances, insistances, ajouts, contradictions...

\section{Cing figures de pratiques SPATIALES SELON LE RAPPORT À LA DURABILITÉ}

La construction de figures n'est ni œuvre de catégorisation ni volonté d'ériger une typologie dans laquelle on ferait entrer les populations étudiées. Il s'agit plus d'une représentation schématique conçue pour illustrer notre propos. Les objets mobilisés et

\footnotetext{
5. Le seul tracé GPS ne fournit pas le motif précis du déplacement mais, replacé sur un fond de carte permettant de connaître les fonctionnalités des lieux, il est aisé, avec une marge d'erreur minime, de saisir le motif de déplacement : travail, loisir, achat, retour au domicile... Au besoin, en cas d'incertitude, il a été possible de demander à la personne, lors de la phase d'entretien, de le préciser.

6. Cette troisième partie du discours, qui touche véritablement la valeur de durabilité, est contrainte par les deux premiers temps et notamment l'épreuve de vérité cartographique, empêchant ainsi la personne de chercher à se valoriser de façon exagérée. La sauvegarde de la cohérence globale s'impose au désir de valorisation personnelle.
} 
les réalités sont trop complexes pour se laisser enfermer dans une simplification qui leur ôterait l'essentiel du sens que l'on y cherche. Toutefois, c'est bien de manière inductive, c'est-à-dire suggérée par les entretiens, que se dégagent cinq figures « d'habitants » en fonction des modalités selon lesquelles ils considèrent la durabilité dans leurs pratiques spatiales et des modes de justification énoncés. Ces figures permettent de cerner les différentes postures avec lesquelles chaque individu jongle à des degrés variables pour justifier ses pratiques et de saisir l'essentiel, c'est-à-dire de quelle(s) manière(s) la durabilité est pensée et pratiquée par les individus.

Premièrement, nous avons le «consommateur». Le maintien de son mode de vie et sa propension marquée à ne pas être sensible à la nécessité de changement auquel l'invite la société et les politiques, sont deux lignes directrices de son profil. Le mode de vie pratiqué est jugé comme «bon » et les mobilités sont pensées en termes d'efficacité, de confort, de facilité et d'intérêt : il ne se pose pas de questions quant aux conséquences éventuelles de son action, par exemple en termes de pollution, ni ne se réfère à un principe qui porterait sur une attention porté à l'environnement ou telle autre dimension de la durabilité. Plus généralement, pour le représentant de cette figure, la réflexion sur son mode de vie et sur la nécessité d'adopter une réflexivité vis-à-vis de son habiter est perte de temps et évacuée. C'est ainsi un nombre non négligeable de personnes qui dénigrent l'idée même de durabilité. Il en découle que toute modification des pratiques - si cette modification n'apporte pas un surcroît d'efficacité ou de confort, ou, au minimum, pérennise la situation dans ses grandes lignes, que cela soit commandé ou non par un principe normatif de durabilité - est perçue comme source de contraintes personnelles; elle est ignorée voire bannie. Pour cette figure, le moteur du changement n'est envisageable qu'à la double condition qu'il permette d'aller dans le sens de la pérennisation du mode de vie et dans le sens d'une durabilité accrue.

Ensuite, le "pauvre » et la notion d'effort renvoient à des individus qui ne disposent pas de facilités d'ordre économique et/ou de redéfinition de leur mode de vie, à même d'inscrire leurs mobilités dans un cadre durable. Ils développent alors un sentiment de culpabilité au regard de leurs pratiques tout en revendiquant, parfois, une opinion positive et favorable au «développement durable ». L'une des personnes enquêtées, M1, nous dit : «Tu vois, maintenant j'ai un peu moins mauvaise conscience si je fais $50 \mathrm{~km}$ pour aller à une fête dans le sud Touraine, mais c'est vrai qu'au début ça me semblait hallucinant de prendre ma voiture toute seule et puis juste pour faire un aller-retour quelque part et...alors bon il $y$ a une espèce de culpabilisation à la con mais quand même moi c'est vraiment cette idée que la bagnole, ça pollue trop quoi. » Cette culpabilité a un corollaire : la notion d'effort. Pour les individus représentatifs de cette figure, le changement, nécessitant un effort jugé considérable, ne peut relever que de l'incitation, généralement financière. Celle-ci peut ainsi apparaître comme un des moteurs possibles du changement car ces individus - cherchant une certaine reconnaissance sociale et leur inscription dans une société changeante et normative - adhèrent et mettent en œuvre les pratiques ne demandant pas d'efforts de cette sorte. On pourrait aller jusqu'à considérer qu'un «terreau » favorable est présent.

« L'écolo » et le militantisme à l'épreuve de son « faire avec » renvoient d'abord à la revendication d'une forte sensibilité au durable. Elle se traduit dans une large gamme de pratiques et - mais il faut sans doute y voir un effet de l'entretien - particulièrement sur la question des transports en commun. Cependant, « l'écolo » doit concilier cette revendication (qui est aussi revendication d'une certaine image de soi en accord avec le sentiment d'être dans la norme, du moins dans une certaine norme) avec la réalité de l'offre territoriale. Le heurt, lié à la différence entre le fait et l'idéal, s'exprime par les contradictions entre la cartographie GPS et le discours tenu. C'est le cas, par exemple, d'une responsable d'association de défense et de promotion de la pratique du vélo qui tient un discours écologiste mais utilise systématiquement sa voiture pour aller au travail alors même qu'un mode de transport en commun efficace existe sur son trajet. Pour cette figure, le «terreau » est bien présent, les moteurs pourraient être l'incitation ainsi que la valorisation personnelle et sociale de l'image de soi.

Pour le «dogmatique » et ses principes à l'épreuve de son «faire avec », l'ensemble de valeurs, érigé en système, qui sous-tend ses pratiques, le conduit à requestionner, à évaluer ses propres pratiques, grâce à une capacité de forte réflexivité. Les représentants de cette figure ont une idée précise de leurs actes et 
de leur situation et considèrent sans concession leur mode d'être et leur mode d'habiter, leur donnant un «sens moral », qui renvoie au bon sens et non à l'image de soi vue par soi-même et secondairement par autrui. Le moteur du changement pourrait s'intégrer dans l'idée d'éducation aux bonnes pratiques sous-tendue par une forme de rationalité éthique (c'est bien d'agir ainsi, parce que...).

Enfin, la figure du «né avant la guerre » s'appuie sur l'idée de réduction du gaspillage. Elle ne se résume pas aux personnes qui auraient nécessairement soixante-quinze ans ou plus. Pour elles, toute pratique est passée au filtre du gaspillage. Cette posture renvoie au bon sens mais elle est d'abord de l'ordre du réflexe, des habitudes acquises ou héritées et qui sont à transmettre comme mode de vie relevant de l'évidence, par opposition à la notion d'effort évoquée précédemment. J6 dit ainsi que ne pas gaspiller est «un sens élémentaire de la responsabilité ». Une autre (M1) qu'elle a "toujours été élevée dans cette idée que l'énergie a un coût, qu'on ne gaspille pas, que, voilà, à un moment donné on essaie de vivre en accord avec ce qu'il y a autour de nous ".

L'élaboration des figures permet de considérer la durabilité non plus seulement comme une contrainte à laquelle il faudrait s'astreindre mais aussi comme une opportunité permettant de réaliser des aspirations personnelles et identitaires. Elles mettent en évidence l'existence de tendances qui reflètent la capacité à s'adapter ou non à un système changeant. Ainsi, les individus s'inscrivent dans deux grands types : ceux qui, absolutistes et déontologiques, tendent vers un mode de vie géré par des principes; ceux qui, relativistes et conséquentialistes, tendent vers un mode de négociation. Ces deux tendances renvoient aux oppositions entre « valeur et effort » d'un côté et «culpabilité et dédouanement » de l'autre, qui s'agencent chez certains de façon très manichéenne ou, pour d'autres, au contraire, de manière plus complexe, dans la justification des pratiques.

En effet, si le " dogmatique » et le " né avant la guerre "se réfèrent uniquement à des valeurs, le «pauvre », qui convoque la notion d'effort et «l'écolo » qui, lui, ne le fait pas, mobiliseront tous deux culpabilité et dédouanement avec plus ou moins de subtilité. Pour le "pauvre », la culpabilité sera celle de n'être pas dans la norme et le dédouanement, vis-à-vis de cette a-normalité, se fera par certaines actions peu coûteuses, et seulement celles-là, pour pouvoir se dire durable. En ce qui concerne "l'écolo », la culpabilité, bien que non affichée, est présente dans la quête de performance; le dédouanement perpétuel également, dont il fait preuve pour se conformer au durable, tel que la société le définit à un instant $\mathrm{t}$.

\section{LA PLACE DE LA VALEUR DE DURABILITÉ}

Ces figures prennent en compte la durabilité des pratiques autant comme une valeur que comme une norme plus ou moins intégrée, plus ou moins acceptée. La durabilité comme valeur renvoie au débat évoqué précédemment entre déontologie et conséquentialisme quant à l'attitude éthique des individus : les justifications données dans l'épreuve de vérité qu'est l'entretien renvoient-elles plutôt à l'une ou à l'autre des deux types d'éthiques?

Tout l'apport éthique de Kant repose sur un double objectif : qu'est-ce qu'une action morale et comment savoir si une action effectuée ou prévue peut être qualifiée de morale? La première renvoie au questionnement philosophique, dont l'essentiel est présenté dans les Fondements de la métaphysique des mœurs, la seconde au questionnement anthropologique. La recherche menée est bien de l'ordre de l'anthropologie, mais la philosophie kantienne fournit le cadre d'interprétation. Selon Kant, la moralité d'une action ne dépend pas du résultat de celle-ci : si une action est intégralement, indubitablement et objectivement durable, elle n'en est pas pour autant automatiquement respectueuse de la valeur de durabilité. Pour l'auteur, la moralité d'une action dépend de l'intention première qui y a présidé et seul le respect de la maxime indiquant le devoir permet de juger éthiquement d'une action. Ainsi, il distingue les actions respectueuses du devoir de celles qui ne sont que conformes au devoir. Dans notre champ d'analyse, nous pouvons, par application de ce principe, proposer une différenciation entre pratiques « objectivement» durables : d'une part celles faites par souci de la durabilité et d'autre part celles conformes à la valeur de durabilité, mais qui auraient été faites pour d'autres raisons. Pour être complet, il faut ajouter aussi les pratiques non conformes - et donc non respectueuses - de la valeur durabilité : celles qui ne sont pas durables. 
La différence entre les actions seulement conformes et celles qui sont aussi respectueuses de la valeur durabilité (au-delà du fait que toutes deux répondent « objectivement » aux critères de durabilité) réside dans le mécanisme de délibération : une action est seulement conforme à la durabilité si sa mise en œuvre découle d'autres critères que la seule durabilité. Ainsi, un individu prenant les transports en commun plutôt que sa voiture pour des raisons liées à son porte-monnaie fait une action seulement conforme au durable. En revanche, s'il prend les transports en communs plutôt que sa voiture pour réduire les émissions de gaz à effet de serre, c'està-dire par prise en compte de la valeur durabilité, son action sera non seulement conforme mais aussi respectueuse du durable.

Qu'en est-il de nos interviewés? «Il est absolument impossible d'établir par expérience avec une entière certitude un seul cas où la maxime d'une action d'ailleurs conforme au devoir ait uniquement reposé sur des principes moraux et sur la représentation du devoir » (Kant, 1988, p. 112) et non, aussi, sur des motivations autres ${ }^{7}$. Cette posture radicale, absolutiste relativement aux valeurs et notamment aux valeurs morales, renforce la détermination du chercheur analysant les dires recueillis afin de mettre au jour, autant que possible, la ou les raisons de telle modalité d'action, pour séparer les mobiles déontologiques des mobiles issus des inclinations, et ainsi estimer si la durabilité est mobilisée comme valeur ou si un autre moteur de l'action est à l'œuvre.

Si l'on relativise le propos kantien, en faisant de l'être moral (déontologique) un idéal-type utilisé comme référence, les actions « relativement respectueuses » de la durabilité seront celles qui, conformes à la durabilité, pourront être supposées faites malgré les bonnes raisons qu'avait l'auteur de l'action de faire autrement (Boudon, 2003). Les actions simplement conformes à la durabilité, qui ne sont donc pas respectueuses de celle-ci, seront

\footnotetext{
7. Cette impossibilité réside dans l'absolutisme même du propos kantien. S'il est aisé d'avoir un doute quant à la moralité d'une action faite par une personne ayant intérêt à faire cette action, il est impossible de connaître les ressorts psychologiques de quelque ordre que ce soit permettant d'assurer à un observateur extérieur (et même à la personne elle-même) que telle action conforme n'a pas été fait, in fine, pour des raisons autres. Dans le cas de quelqu'un qui agit à l'encontre de ses intérêts, malgré ceux-ci, le respect de la valeur est possible mais toujours douteux. Dans le cas de quelqu'un qui agit dans le sens de ses intérêts, a-t-il la possibilité de faire abstraction de ceux-ci pour n'être motivé que par le respect qu'il porte à la maxime de son action? Et que peut en dire un observateur extérieur?
}

celles qui auront été justifiées, dans les discours par une ou plusieurs bonnes raisons qui ne relèvent pas du respect de la durabilité. En termes de résultats, il faut bien considérer que les actions conformes, aussi nombreuses soient-elles, ne peuvent être que très rarement considérées comme respectueuses au sens kantien du terme. À l'échelle d'un individu (c'est-à-dire de ses actions décrites), on peut effectivement considérer que certaines personnes sont plus respectueuses que d'autres de la durabilité mais, là aussi, qu'une référence strictement morale est quasi inexistante.

Au-delà des attitudes décrites précédemment, on constate deux grands types de comportement : la conciliation et la compensation. Toutes deux sont ancrées dans l'idée d'un relativisme des valeurs qui éloigne l'essentiel des dires recueillis d'une conception strictement kantienne de l'éthique. Mais cela n'évacue pas complètement l'idée de la durabilité comme principe, même si ce principe n'est pas toujours appliqué. Il est plus souvent négocié en fonction d'attentes autres, c'est-à-dire avec des actions non durables ou simplement conformes au durable. Ces négociations montrent que l'individu lambda ne se heurte généralement pas aux contradictions inhérentes aux dilemmes moraux liés au jusqu'auboutisme du déontologique ou celui du conséquentialisme décrits par exemple par Tappolet (1996) ou Ogien (2011).

La première, la conciliation, est une délibération que fait l'individu en lui-même. S'il peut prendre en considération des éléments relatifs à d'autres personnes, c'est toujours du point de vue du délibérant. Il n'est pas dans une phase de négociation, sinon avec lui-même. Cette délibération est relative à une situation donnée, à un acte (et un seul) et aux modalités de sa mise en ouvre : objectif à atteindre, contraintes, délai et/ou urgence, choix des possibles... tels que considérés par la personne. La délibération se fait "sur le vif », elle est intuitive, de l'ordre de l'instantané. Les conséquences sont très peu examinées. Cette conciliation est aussi le résultat de la confrontation de valeurs qui président à l'action et des conséquences de cette action, pour soi-même ou plus largement. Dans nombre de cas, c'est l'habitude ou la routine qui préside à la mise en œuvre d'un acte. On peut considérer cependant que le « début » d'une habitude fait l'objet d'une délibération de type conciliation. 
La compensation s'oppose, sur certains points, à la conciliation tout en la complétant. Le discours de justification des pratiques ne peut faire l'économie d'une relation dialogique entre conciliation et compensation. Alors que la conciliation se fait relativement à un acte, la compensation s'effectue nécessairement entre plusieurs actes, entre plusieurs catégories d'actes : à l'intérieur d'un même poste (les déplacements par exemple) ou en mettant en balance plusieurs postes (déplacements, consommation alimentaire, isolation et mode de régulation de la température du logement...). De ce fait, alors que la conciliation, se faisant «sur le vif », est du domaine de l'action, de l'acte, la compensation dépasse celui-ci. Elle s'intègre dans un ensemble plus large, ensemble dont les constituants sont choisis de façon non neutre, à la fois en fonction de données objectives et en fonction du résultat que la personne attend de cette délibération, l'autorisant ou non à justifier tel acte, tel type d'actes ou telle série d'actes. La compensation se fait selon deux grands modes que l'on peut présenter ainsi : " ayant "bien" agi, je peux maintenant me permettre d'agir "moins bien" » ou bien "ayant pris conscience de la nature et des conséquences de mes actions, qu'au final j'évalue négativement, j'essaie de me "rattraper" ». L'idée de compensation renvoie à celle de bilan, bilan nécessairement partiel, relatif à une partie de la vie de l'individu. Si ce bilan peut se faire à n'importe quel moment, notamment quand se fait jour aux yeux de l'individu un décalage trop grand entre ce qu'il souhaiterait faire pour être cohérent avec ses valeurs et ce qu'il fait réellement, l'enquête est un moment privilégié pour faire ce bilan, le conscientiser et, par l'entremise de cette compensation, donner à la fois une justification globale à l'ensemble de ses actes et une justification à chacun d'eux, si l'épreuve de vérité que représente ce moment le conduit ou l'oblige à aller dans le détail.

Deux points paraissent devoir être soulignés. Du côté des conciliations, l'individu doit bien être compris comme étant à la fois capable et obligé de concilier, de façon quasiment continuelle. Comme la situation n'est jamais optimale, seulement plus ou moins satisfaisante, il faut faire avec elle, parce que les individus ne peuvent pas ne pas avoir certaines pratiques - tant celles liées au travail qu'aux diffé- rentes sphères ${ }^{8}$ mises en avant - et parce qu'ils ont des valeurs ou du moins des éléments de références (valeurs, exemples, modèles, habitudes) sur lesquels ils assoient leurs pratiques. Les individus sont amenés à concilier sans cesse ce qu'ils font (et comment ils le font) avec ce qu'ils voudraient faire (et comment?) et avec ce qu'il leur est possible de faire. Ils concilient leurs souhaits avec ceux des autres, leurs attentes et ce que leur permet le territoire. Ils concilient les temps impartis à chaque activité, y compris celle de se déplacer. Plus encore, la conciliation, telle que nous l'avons élaborée conceptuellement, dépasse en la précisant l'idée de «faire avec ». Elle est un "faire avec » multiple dans la mesure où elle fait avec de nombreux éléments de l'espace, y compris contradictoires. Elle englobe les différents possibles que propose le territoire, qu'elle croise avec ce que veut et peut l'individu.

Face aux diverses injonctions au durable émises par la société, face aux injonctions que la personne se fait à elle-même, face au problème insoluble d'une rencontre " parfaite » entre l'idéalité des valeurs et leur mise en pratique dans un environnement changeant et contraignant, la compensation est, de son côté, la recherche d'un équilibre. Cet équilibre est recherché pour que la personne puisse se reconnaître dans ses valeurs en même temps que dans l'application de celles-ci dans ses propres pratiques, le tout dans un contexte qu'elle ne maîtrise pas. En effet, son système de valeurs qui sous-tend toutes ses actions, généralement de façon implicite, est sans cesse contredit par les contraintes, par la réalité des faits. Ainsi, la compensation permet-elle que l'image que la personne a d'elle-même soit suffisamment positive pour que, à une échelle temporelle longue et sur des champs de pratiques différents, elle atteigne cet équilibre, changeant et instable.

\section{Conclusion}

La durabilité, comme notion, poursuit son chemin : de concept politique elle devient norme

\footnotetext{
8. La recherche Périvia avait structuré les justifications autour de « sphères »: intime (différenciation, particularité, sentiment, habitude), domestique/ familiale (premier groupe d'appartenance/contrainte ou liberté), sociétale (travail, ville, environnement social), temporelle (échelle de la quotidienneté, agenda et gestion des temps), spatiale (distance, petit, grand, paysage, esthétique), technique (mode de déplacement, modalité de la mobilité, performance), normative (des normes et représentations sociales aux valeurs personnelles).
} 
technique et injonction sociale. Parallèlement, elle devient valeur. En tant que telle, quelle que soit la définition que l'on mobilise de la valeur, elle teinte une partie, plus ou moins importante, des activités des individus, que ce soit dans le champ des pratiques spatiales ou plus largement dans l'habiter des individus, leur rapport à l'espace, que l'on observe simplement ces pratiques ou qu'on en attende la justification éthique. Une autre étude (Hakim, 2011) a pu, par un protocole complètement différent, mettre en évidence le statut de valeur de la durabilité. Elle a montré que l'indication du caractère durable d'un bâtiment joue positivement sur son évaluation esthétique.

Que ce soit par le biais de la sensibilisation, celle des enfants et adolescents via le système scolaire, ou celle de tout citoyen, par le biais de l'enchérissement de l'énergie ou de la diminution relative des moyens financiers, la durabilité est devenue valeur, non au sens strictement kantien du terme, mais davantage (pas uniquement) au sens conséquentialiste et relativiste de celui-ci. Cette valeur teinte donc aujourd'hui le rapport à l'espace des individus, leur mode d'habiter, tant en termes de pratiques de déplacement que d'activités localisées, même si bien d'autres considérations doivent être prises en compte (aspects purement financiers, image de soi, routines, méconnaissance du champ des possibles, méconnaissance de la totalité du champ des conséquences des activités...). C'est la composition des justifications apportées aux mobilités qui montre l'imbrication entre déplacements et localisations, dans les choix quotidiens qui sont faits pour donner corps à chacun des moments et sens à l'ensemble qu'ils forment.

\section{Bibliographie}

Aristote, 1992. L'éthique à Nicomaque, Le Livre de poche, Paris, $448 \mathrm{p}$.

Aubertel P., Bonnet M. (dir.), 2006. La ville aux limites de la mobilité, Paris, PUF, 316 p.

Bailleul H., Feildel B., 2011. Le sens des mobilités à l'épreuve des identités spatiales : un éclairage par le récit de vie spatialisé et l'herméneutique cartographique, in DEPEAU S., Ramadier T. (dir.), Se déplacer pour se situer. Places en jeux, enjeux de classes, Rennes, PUR, p. 25-55.

Bassand M., Brulhardt M.-C., 1980. Mobilité spatiale, SaintSaphorin (Lavaux), Ed. Georgi, 300 p.
Bentham J., 1970. An Introduction to the Principles of Morals and Legislation, The Althone Press, London.

Bosc Ch., 2003. Emergence et négociation de politiques environnementales locales à Lyon et Montpellier, Thèse de Doctorat, Université de Montpellier.

Boudon R., 2003. Raison, bonnes raisons, Paris, PUF.

Cailly L., 2007. La diversité des modes d'habiter des espaces périurbains dans les villes intermédiaires : différenciations sociales, démographiques et de genres, Norois, vol. 4, $n^{\circ} 205$, p. 67-80.

Cailly L., 2008. Existe-t-il un mode d'habiter spécifiquement périurbain?, EspacesTemps. net, 13.05.2008, [http://test. espacestemps.net/articles/existe-t-il-un-mode-drsquohabiterspecifiquement-periurbain/], consulté le 25 janvier 2013.

Carpentier S., 2007a. Une analyse exploratoire des liens entre mobilité quotidienne et ancrage résidentiel : vers une approche écologique de la mobilité?, Articulo.ch, revue de sciences humaines, $\mathrm{n}^{\circ} 3$.

Carpentier S., 2007b. Mobilité quotidienne et ancrage résidentiel. Différenciation des pratiques spatiales et des représentations sociales selon la structure urbaine : l'exemple $d u$ Luxembourg, thèse de doctorat en géographie, université Strasbourg I, 392 p.

Chardonnel S., 2011 . «Projet TRACES : évaluation comparée de l'apport de l'assistance GPS aux enquêtes de mobilité », Communication au séminaire UrbanFabrik, Grenoble, 7 décembre 2011.

Cordobes S., Lajarge R., Vanier M., 2010. Pour des périurbains assumés, in DATAR, Territoires 2040, DATAR, Paris, p. 22-32.

Courgeau D., 1988. Méthodes de mesure de la mobilité spatiale, INED, Paris, $301 \mathrm{p}$.

Da Cunha A., 2005. Régime d'urbanisation, écologie urbaine et développement urbain durable : vers un nouvel urbanisme, in Da Cunha A., Leresche J.-P., Knoepfel P., Nahrath S. (dir.), Enjeux du développement urbain durable, Lausanne, Presses Polytechniques et Universitaires Romandes, p. 12-38.

Depeau S., 2011. «À la recherche des espaces invisibles de la mobilité : usage, apports et limites des techniques GPS dans l'étude des déplacements urbains à l'échelle pédestre ", Communication au séminaire UrbanFabrik, Grenoble, 7 décembre 2011.

Dodier R. (dir.), 2007. Vivre les espaces périurbains, Norois, numéro spécial, vol. 4, n²05, 136 p.

Dodier R., 2009. Individus et groupes sociaux dans l'espace, apports à partir de l'exemple des espaces périurbains, mémoire d'Habilitation à Diriger des Recherches, vol 3, université du Maine, 244 p., [http://tel.archives-ouvertes.fr/tel-00430480].

Dureau F., LÉvy J.-P., 2002. L'accès à la ville. Les mobilités spatiales en question, Paris, L'Harmattan, 412 p.

EhEA, 2008. Rapport final, Espaces habités et espaces anticipés: qualification de l'espace, ANR, [http://citeres.univ-tours.fr/ compo.php?niveau=ipapeEpage $=$ p_ipape/ipape_online $]$.

Emelianoff C., 2007. La ville durable : l'hypothèse d'un tournant urbanistique, L'Information géographique, vol. 71, n 3 , p. $48-65$. 
Feildel B., Martouzet D., 2012. La mobilité comme modalité de l'ancrage : enrichir l'évaluation de la durabilité des espaces périurbains, Recherche Transports Sécurité, octobre 2012, p. 271-289.

Накім L., 2011. L'influence des valeurs écologiques sur la perception visuelle et l'évaluation esthétique du bâtiment, Mémoire de master, Université de Tours, 161 p.

Kant E., 1988, Fondements de la métaphysique des mours, Paris, Delagrave, $210 \mathrm{p}$.

Kauffman V., 2008. Les paradoxes de la mobilité : bouger, s'enraciner, Lausanne, Presses polytechniques et universitaires romandes, $115 \mathrm{p}$.

Kaufmann V., 1999. Mobilité et vie quotidienne : synthèse et questions de recherche, 2001 Plus, n 48, 63 p.

Keramidas O. et al., 2010. Ethique et durablité : quelle construction, quelles analyses, quelles perspectives?, Journée Développement Durable de l'AIMS [http://www.cergam. org/fileadmin/files/cerog/wp/880.pdf], consulté le 25 janvier 2013.

LÉvy J., 2003. Géotype, in LÉvy J., Lussault M., Dictionnaire de la géographie et de l'espace des sociétés, Paris, Belin, 1033 p.

Livet P., 2006. Les normes, Paris, Armand Colin, 168 p.

Mancebo F., 2007. Quels référentiels pour un aménagement "durable" ?, L'Information géographique, n 3, p. 29-47.

Martouzet D., Bailleul H., Feildel B., 2010. Les justifications de la mobilité périurbaine : mise à l'épreuve de la durabilité dans un espace intermédiaire, in Giroud M., Mainet H., Edouard J.-C., Les mobilités spatiales dans les villes intermédiaires, Clermont-Ferrand, Presses Universitaires Blaise-Pascal, p. 11-30.

Marzloff B., Kaplan D., 2009. Pour une mobilité plus libre et plus durable, Paris, Ed. FYP, 86 p.

Mathieu N., Guermond Y., 2005. La ville durable : un enjeu scientifique, in Mathieu N., Guermond Y. (dir.), La ville durable, du politique au scientifique, Paris, Cemagref Editions, Cirad, Ifremer, INRA Editions, p. 11-29.

Montulet B., 1998. Les enjeux spatio-temporels du social: mobilités, Paris, L'Harmattan, 220 p.

Navez-Bouchanine F., 2007. Le développement urbain durable : "best practice" ou leurre méthodologique, Espaces et sociétés, vol. 4, $\mathrm{n}^{\circ} 131$, p. 101-116.

Ogien R., 1996. Normes et valeurs, in Canto-Sperber M., Dictionnaire d'éthique et de philosophie morale, Paris, PUF, p. 1052-1064.

OGIEN R., 2011. L'influence de l'odeur des croissants chauds sur la bonté humaine et autres questions de philosophie morale expérimentale, Paris, Grasset.

Orfeuil J.-P., 1998. Dis-moi où tu habites, je te dirai comment tu te déplaces, in Pumain D., Mattei M.-F., Données urbaines, Paris, Anthropos, p. 157-164.
Paquot T, Lussault M., Younès C., 2007. Habiter, le propre de l'homme. Villes, territoires et philosophie, La Découverte, Paris, 379 p.

Pharo P., 2007. Valeurs, in Encyclopaedia Universalis, dictionnaire de sociologie, Paris, Albin Michel, p. 835-839.

RAmadier T. et al., 2007. Rapport final. Les mobilités quotidiennes : représentations et pratiques. Vers l'identité de déplacement, ATIP, n 41799.

Ramadier T., Lannoy P., Depeau S., Carpentier S., Enaux C., 2009. Vers l'hypothèse d'une identité de déplacement. Congruence entre espace social, cognitif et géographique, in Grandjean P. (dir.), Construction identitaire et espace, Paris, L'Harmattan, p. 75-94.

RÉmy J., 1996. "Mobilités et ancrages : vers une autre définition de la ville », in Hirschhorn M., Berthelot J.-M., Mobilités et ancrages. Vers un nouveau mode de spatialisation, Paris, L'Harmattan, p. 135-153.

Roux E., VAnier M., 2008. La périurbanisation : problématiques et perspectives, La Documentation française, Paris, 87 p.

Salomon Cavin J., Bourg D., 2010. Deux conceptions de la durabilité urbaine : ville prométhéenne versus ville orphique, in Paquot T., Younès C. (dir.), Philosophie de l'environnement et milieux urbains, Paris, La Découverte, p. 117-136.

STоск M., 2005. Les sociétés à individus mobiles : vers un nouveau mode d'habiter?, EspacesTemps. net, 25.05.2005, [http://www.espacestemps.net/articles/les-societes-a-individusmobiles-vers-un-nouveau-mode-drsquohabiter/].

Sтоск M., 2006. L'hypothèse de l'habiter poly-topique : pratiquer les lieux géographiques dans les sociétés à individus mobiles, EspacesTemps. net, 26.02.2006, [http://www.espacestemps.net/articles/lrsquohypothese-de-lrsquohabiter-polytopique-pratiquer-les-lieux-geographiques-dans-les-societes-aindividus-mobiles/].

Tappolet C., 1996. Dilemmes moraux, in Canto-Sperber M. (dir.), Dictionnaire d'éthique et de philosophie morale, Paris, PUF, p. 417-423.

Thévenot L., 1996. Justification et compromis, in Canto-SperBER M. (dir.), Dictionnaire d'éthique et de philosophie morale, PUF, Paris, 1996, p. 789-794.

URRY J., 2005. Sociologie des mobilités : une nouvelle frontière pour la sociologie?, Paris, Armand Colin, 253 p.

Villalba B., Lejeune C., 2011. La durabilité en renfort. Requalification d'un projet urbain. Le cas de la Zone de l'Union (Nord, France), Cosmopolitiques, n 19, mai 2011, Dossier « Les Ecoquartiers », [http://www.cosmopolitiques. com/], consulté le 25 janvier 2013.

Weber M., 1959, Le savant et le politique, Paris, Plon, 184 p. 